\title{
Performance and Stability Assessment of Future Grid Scenarios for the Australian NEM
}

\author{
Hesamoddin Marzooghi ${ }^{1}$, Student Member, IEEE, David J. Hill ${ }^{1,2}$, Fellow, IEEE, \\ and Gregor Verbic ${ }^{1}$, Senior Member, IEEE \\ ${ }^{1}$ School of Electrical and Information Engineering, The University of Sydney, Sydney, New South Wales, Australia \\ Emails:\{hesamoddin.marzooghi, david.hill, gregor.verbic\}@ sydney.edu.au \\ ${ }^{2}$ Department of Electrical and Electronic Engineering, The University of Hong Kong, Hong Kong \\ Email: dhill@eee.hku.hk
}

\begin{abstract}
Shifting towards higher penetration of diverse renewable energy sources (RESs) in power systems is motivated mainly by reducing carbon emissions. In the long term of several decades, which we refer to in terms of the future grid (FG), balancing between supply and demand will become more challenging. Also, displacing conventional generators with RESs, especially inverter-based and intermittent RESs, could have significant effects on performance and stability of FGs. So far, FG feasibility studies have mostly considered simple balancing, but largely neglected network related issues such as line overload and stability. The main contribution of this paper is to present a simulation platform for performance and stability assessment of FG scenarios. As a case study, preliminary results on the balancing and stability of the Australian National Electricity Market in 2020 are illustrated with the increased penetration of wind and solar generation in the grid. Simulation results illustrate the importance of power system stability assessment for FG feasibility studies.
\end{abstract}

Keywords-Balancing, electricity market, future grids, power system stability, renewable energy sources

\section{INTRODUCTION}

In conventional power systems, large thermal power plants have provided the main balancing and stability control. Over the past decade, however, there has been a significant deployment of renewable energy sources (RESs) in the world due to environmental issues. Australia's renewables portfolio target aims at increasing the penetration of RESs to $20 \%$ and $50 \%$ by 2020 and 2050, respectively [1]. Consequently, maintaining the balance between supply and demand will become more challenging with the increased penetration of intermittent RESs in future grids (FGs). Also, this change will most likely influence performance, stability and security of the power systems in ways that have not been experienced. So, it is highly important to ensure that power system dynamics are within bounds and stable (i.e. for angle, voltage and frequency), after basic balancing studies for FG scenarios. The conventional power system models, which are well-known and standardised, will need to be augmented by all the new features of FGs (e.g. RESs, demand-side control, etc.). Therefore, the role of modelling and analysis related to balancing and security for FG scenarios remains of central importance.

The literature review below considers studies which relate directly to meeting the new modelling and analysis challenges in FGs [2]-[8]. A study by the University of Melbourne Energy Research Institute has proposed a zero-carbon electrical grid for Australia in 2020 [2]. The aim of that proposal is to provide an effective and efficient grid for the future of Australia relying $100 \%$ on RESs. However, lack of performance and stability assessment of the proposed network makes the proposal highly speculative. The University of New South Wales researchers have analysed the viability of $100 \%$ RES scenarios considering a copper plate model for the Australian National Electricity Market (NEM) [3], [4]. Those studies have shown that relying $100 \%$ on RESs for the NEM can be technologically feasible within the specified NEM reliability standard. Also, the leastcost mix of $100 \%$ RES scenario including wind farms (WFs), utility photovoltaic (PV), concentrated solar plants (CSPs) with storage, hydro and biofuelled gas turbines (GTs) has been determined. Researchers in the USA have proposed a combination of RESs (i.e. onshore and offshore WFs, utility PV and fuel cells) and conventional generation (i.e. GTs) for the future of the PJM network considering a copper plate transmission network [5]. They have shown that the PJM network can be powered $90-99.9 \%$ of the time entirely on RESs, at a cost comparable to today's. Similarly, the leastcost mix of RESs (i.e. WFs, CSPs, utility PV, hydro and geothermal) and conventional generation (i.e. GTs) has been determined for California for 2050 in [6]. While 100\% RESs may or may not be realistic, what is inescapable is that to reverse climate change (without the risk of nuclear power) we need at least triple RES (energy) [7].

The existing studies have demonstrated that aggressive reduction in fossil fuel use is possible, and provides a vision for FGs. However, they have only focused on balancing and neglected the network aspects by using a copper plate model. This assumption can influence the results of FG feasibility studies significantly. The only study that has so far considered stability aspects is a German study [8] that included voltage and frequency stability. The study has shown that for the considered generation mix, voltage and frequency stability deteriorate for some operating points. Due to the stability issues, they have debated that network expansion [8] and additional services by RESs (such as voltage and frequency support) [9] will be required in the German FG.

The main contribution of this paper is to present a simulation platform for performance and stability assessment of FG scenarios. The simulation platform considers market simulation, load flow calculation and stability assessment together. As a case study, the effect of increased penetration of WFs and CSPs on the balancing, performance and stability of the 
NEM is studied using a modified 14-generator model [10]. The electricity market model is built in PLEXOS based on the suitably modified 14-generator model, and the dispatch results from the market are used for balancing and stability studies. Three scenarios are considered: (i) in the business as usual (BAU) Scenario in 2020, the electricity supply is dominated by coal, gas, hydro, and biomass; (ii) in the Renewable Scenario-Case A, some of the conventional coal generators in Queensland and South Australia are replaced with CSPs and WFs, respectively; and (iii) in the Renewable Scenario-Case B, further coal generators in Queensland and South Australia are replaced with CSPs and WFs, respectively. Displacement of the conventional generators in the renewable scenarios is inspired by the studies in [2], [11]. Simulation results show the importance of power system stability assessment for FG feasibility studies.

The remainder of the paper is organized as follows: Section II describes the Australian NEM model. Section III describes simulation platform for performance and stability assessment of FG scenarios. Section IV includes simulation scenarios and discusses simulation results, and, Section V consists of the conclusion of the simulations.

\section{The Australian NEM Model}

This paper presents a simulation platform for performance and stability assessment of FG scenarios including (i) market simulation, (ii) load flow calculation, and (iii) stability assessment together which is described in Section III in detail. In this paper, a modified 14-generator model of the NEM, which was originally proposed for small-signal stability studies [10], is used as the test-bed. The following subsections describe the test-bed and market assumptions and modelling.

\section{A. Test-bed assumptions and modelling}

The schematic diagram of the 14-generator model of the NEM is shown in Figure 1. Areas 1 to 5 represent Snowy Hydro (SH), New South Wales (NSW), Victoria (VIC), Queensland (QLD) and South Australia (SA), respectively. The excitation system (AVR) and power system stabiliser (PSS) of generators are adopted from reference [10]. Also, it is assumed that all thermal, gas and hydro power plants have standard steam turbine governor (i.e. IEEEG1), gas turbine governor (i.e. GAST) and hydro turbine governor (i.e. HYGOV), respectively.

The NEM has been split into 16 zones according to the Australian Electricity Market Operator's (AEMO's) planning document to capture differences in generation technology capabilities, costs, weather and so on in the future [11], as shown in Figure 2. The predicted hourly demand data by the AEMO is aggregated across each region of the NEM (i.e. QLD, NSW, VIC and SA). The demand data for each region is divided between the available loads of the 14-generator model in that region (i.e. based on their default values). The modified 14-generator model of the NEM is then modeled in PLEXOS and DIgSILENT PowerFactory for the market simulations, balancing and stability studies, respectively.

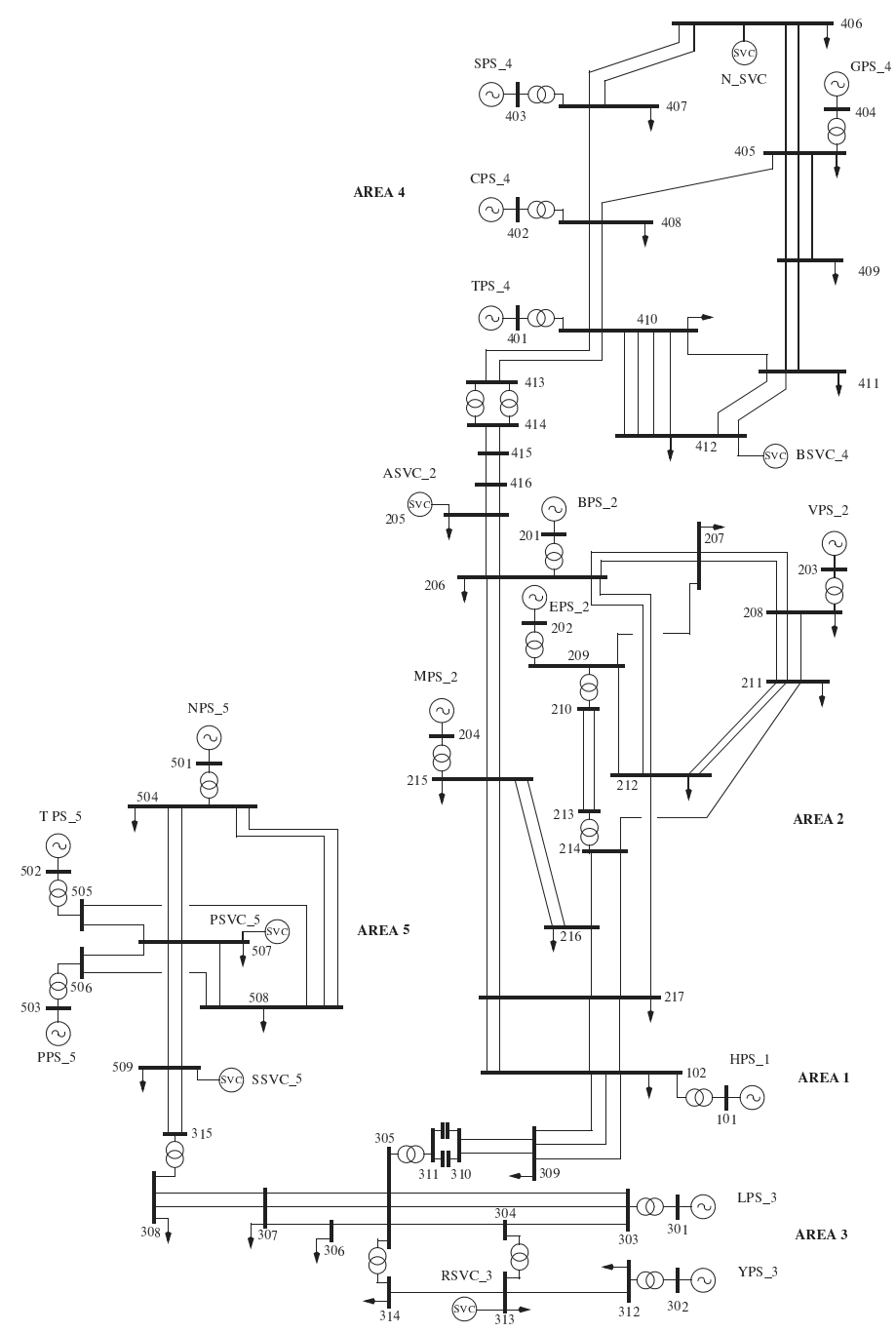

Fig. 1. 14-generator model of the NEM [10]

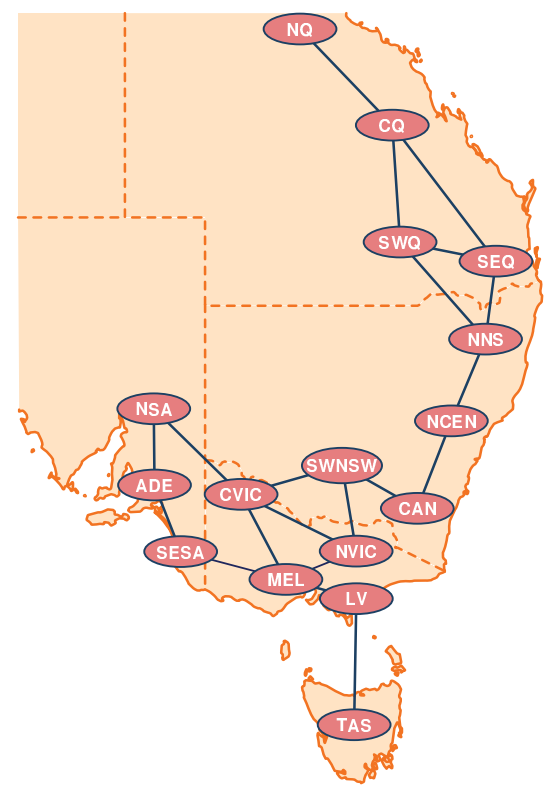

Fig. 2. Proposed 16 zones for the NEM by the AEMO [11] 


\section{B. Electricity market assumptions and modelling}

The market simulations of this paper are done in PLEXOS, effectively mimicking the dispatch process used by the AEMO. The resolution of the market simulations is taken as one hour. Combinations of coal, hydro, biomass and GT are considered for the NEM to supply the load in 2020 in the BAU Scenario. In this paper, CSP controllers are assumed to be similar to thermal power plants. Also, a fully rated converter-based WF model in DIgSILENT which is based on the generic wind turbine model [12] is used here. For each hour of the year, the mixed integer linear solver dispatches the generation, in merit order, to meet demand for that hour. In market simulations, the fossil-fuel generators were assumed to bid at their respective short-run marginal costs (SRMC), calculated using the predicted fuel price, thermal efficiency and variable O\&M in 2020 [11], [13], while the SRMC of renewable generation is assumed to be zero. SRMC for coal and biomass power plants are between $\$ / M W h$ 21-40. Also, SRMC of GT power plants are between $\$ / M W h$ 69-75. Moreover, the interstate line limits are considered for the market simulations according to the NEM limits [11]. The market model also considers the minimum stable level of generators reported in [11].

If supply cannot meet the demand, the hour is recorded as the unmet hour. However, if available generation exceeds demand (i.e. due to high generation of intermittent RESs), the surplus power is recorded as spilled energy and that hour is marked as a spilled hour. Finally, the dispatch results are used as inputs for DIgSILENT for balancing and stability studies.

\section{Simulation Platform}

The simulation platform for performance and stability assessment of FG scenarios is shown in Figure 3. Firstly, FG scenarios have to be generated. In this study, FG scenarios are inspired by the studies in [2], [11], and are described in Section IV in detail. Also, an accurate data source for the electricity demand and weather (e.g. wind, solar irradiance, etc.) is required. In this paper, resolution of demand and weather data is an hour, however, for more detailed studies higher resolution of data might be required. Surprisingly, in some of the existing studies (e.g. [3]-[5]), demand and weather data from the past are used for FG scenarios. This dubious choice can influence the results of FG feasibility studies considerably.

In particular, a significant change in the load profile might be observed in the future due to high penetration of distributed generations and storage [11], [14]. In Australia over 3 GW of rooftop PV generation had been installed mostly by residential and commercial customers until 2013 [15]. Also, installed battery storage capacity has been increasing in the past couple of years [11], [14]. It is predicted that penetration of PV-plusstorage system in Australia will increase further in the next decades [11], [14], and, this will greatly influence the load profile for FG scenarios [14]. Figure 4 shows the aggregated demand profile for the NEM for different PV-plus-storage uptake scenarios of residential and commercial customers from $19^{\text {th }}$ to $22^{\text {nd }}$ of January 2020. This is part of our ongoing research to model aggregated effect of demand-side for FG scenarios.

In this study, preliminary results for performance and stability assessment of FG scenarios for the NEM are presented

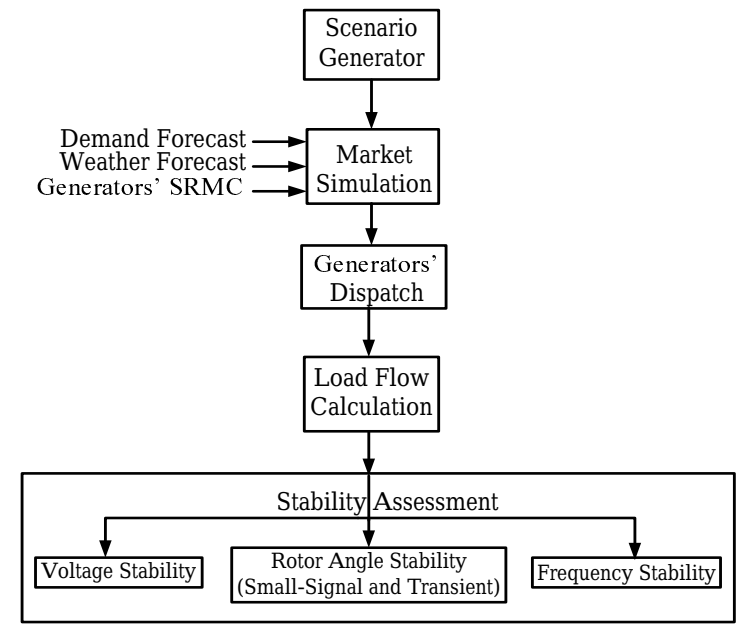

Fig. 3. Simulation platform for performance and stability assessment of FG scenarios

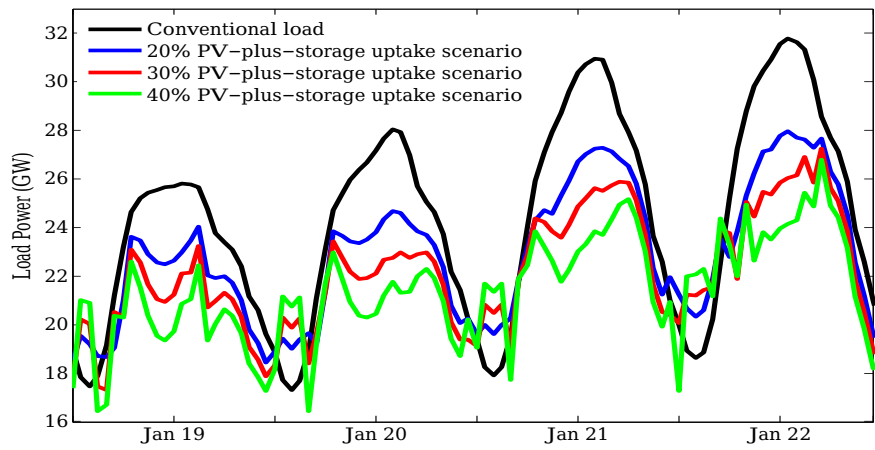

Fig. 4. Aggregated demand profile for the NEM for different PV-plus-storage uptake scenarios from $19^{\text {th }}$ to $22^{\text {nd }}$ of January 2020

using the predicted demand and weather data in 2020 by the AEMO [11]. The following subsections describe the implementation of (i) market simulation, (ii) load flow calculation, and (iii) stability assessment together for FG feasibility studies.

\section{A. Market simulation}

In the first step, market simulation has to be done because dynamics of the market can interfere with the system stability, as anticipated many years ago [16]. In this paper, the resolution of the market simulations is one hour, however, it can be extended (e.g. 5 minutes) for more detailed studies. Also, market constraints have to be considered for FG scenarios (e.g. minimum stable level and ramp rate of generators, etc.), as they can change the dispatch results significantly. PLEXOS is a powerful market simulator which can consider all the market constraints for the dispatch process, and is used in this study as described in Section II. As it can be seen in Figure 3, dispatch results from the market will be used for balancing and stability assessment of FG scenarios.

\section{B. Load flow calculation}

In the second step, the load flow has to be calculated using the dispatch results. The aim of this stage is to be sure that balancing between supply and demand is maintained, and 
also power system variables (e.g. voltage angle and magnitude) are within acceptable bounds at steady-state operation. DIgSILENT is a power system simulator which considers all the limits (e.g. reactive power limit of generators and shunt elements, etc.) and controller actions for load flow calculation, and is used for balancing studies in this paper. The outputs of the load flow will be used as equilibrium and/or initial conditions for power system stability assessment.

\section{Stability assessment}

In the third step, using the information from the load flow, power system stability has to be assessed to be sure that the system can be operated securely after being subjected to disturbances. RESs are displacing conventional generators, and this may affect dynamical behaviour of FGs. Intermittent RESs change dispatch results and operating conditions in the grid (i.e. they are non-dispatchable and bid at zero SRMC). Oscillation modes of the system can be affected because of intermittency and controllers of those sources. On the other hand, inverter-based RESs cannot provide rotational inertia for the grid. So, frequency and transient dynamics might be faster with lower rotational inertia in FGs. Poorly damped oscillation modes might also be experienced because of those sources in power systems. In this paper, different stabilities have been assessed with DIgSILENT using the following methods:

1) Rotor angle stability: In this study, damping ratio of the least stable rotor angle mode in the system is calculated with DIgSILENT using eigenvalue analysis method. DIgSILENT uses QR method [17] for eigenvalue analysis. Also, timedomain simulation with DIgSILENT is used for transient stability assessment of the network. The time frame of interest in transient stability studies is chosen 20 seconds, and, critical clearing time (CCT) is calculated as an indicator for measuring transient stability.

2) Voltage stability: In this study, loadability in the system is evaluated with DIgSILENT. DIgSILENT uses P-V/Q-V method [17] for the loadability calculation of the network. The assumptions for the loadability calculation is described in Section IV.

3) Frequency stability: Time-domain simulation with DIgSILENT is used for frequency stability evaluation of the system in this paper.

\section{Simulation Scenarios AND RESUlts}

The following subsections describe simulation scenarios, and discuss preliminary results on balancing and stability of the NEM in 2020 using the platform in Section III.

\section{A. Simulation scenarios}

As this case study, three scenarios are considered for the NEM in 2020. Displacement of the conventional generators in the renewable scenarios is inspired by the studies in [2], [11]. Also, in all the scenarios, the equivalent MVA rating of the displaced conventional generator is considered for RESs. The description of each scenario is provided in the following:

1) BAU Scenario: In this scenario, combinations of coal, gas, hydro, and biomass are considered for the NEM to supply the load (i.e. zero intermittent RESs penetration).
2) Renewable Scenario-Case A: In this scenario, some of the conventional coal generators in QLD and SA are replaced with CSPs and WFs, respectively. NPS 5 in SA and GPS_4 in QLD are replaced with WF and CSP using NSA and CQ data, respectively. This scenario is called RES-Case A in the rest of the paper.

3) Renewable Scenario-Case B: In this scenario, further coal generators in QLD and SA are replaced with CSPs and WFs, respectively. NPS_5 and PPS_5 in SA are replaced with WFs using NSA and SESA data, respectively. Also, GPS_4 and CPS_4 in QLD are replaced with CSPs using CQ data. This scenario is called RES-Case B in the rest of the paper.

\section{B. Balancing results}

The balancing results for all the scenarios over the simulated year are summarized in Table I. The results show that with the increased penetration of intermittent RESs in the grid, spilled and unmet hours increase from 0 and 0 hours in the BAU Scenario to 462 and 6 hours in the RES-Case B Scenario, respectively. So, with higher penetration of intermittent RESs in FGs, balancing between supply and demand will become more challenging. Due to enough backup supply (i.e. GTs) in the grid, balancing is maintained over $99.9 \%$ of the time in the RES-Case B Scenario. However, it is notable that the required electrical energy from GTs increases from 18.73 TWh in the BAU Scenario to 31.73 TWh in the RES-Case B Scenario. This clearly implies that for higher penetration of intermittent RESs in FGs, higher capacity of backup supply will be required. To keep the backup supply capacity reasonable, demand response (DR) as a powerful resource will likely emerge in the future [18], [19], necessitating the importance of DR modelling at high voltage levels for performance and stability assessment of FG scenarios. The modelling and effect of DR in FG scenarios will be reported separately.

\section{Stability results}

The basic requirement after balancing is maintaining adequate stability margins for power systems. Figure 5 (a) and (b) show demand profile for the NEM and total intermittent RESs generation in renewable scenarios (i.e. RES-Case A and B) form $15^{\text {th }}$ to $18^{\text {th }}$ of January 2020 , respectively. In the following subsections, as an example from the simulated year, stability results are demonstrated for that period of time.

1) Rotor angle stability: Damping ratio of the least stable rotor angle mode improves significantly for many operating points due to increased penetration of WFs in SA, as it is shown in Figure 6. However, under high generation from WFs and low demand in SA, small-signal stability deteriorates and the network becomes unstable. In such a situation dispatch results for the network changes, and generation from the generator(s) in VIC increases because they are cheaper than conventional generators in SA. Increasing the internal rotor angle of generator(s) in VIC participates in small-signal instability for those hours.

In terms of transient stability, based on the operating point, type and location of fault both detrimental and beneficial impacts are observed with the increased penetration of intermittent RESs. In this study, a three-phase fault is considered to be applied at the middle of the line at 1 second. Figure 7 (a) 
TABLE I. BALANCING RESULTS FOR ALL THE SCENARIOS

\begin{tabular}{|c|c|c|c|}
\hline & \multicolumn{3}{|c|}{ Scenarios } \\
\hline Balancing results & BAU & RES-Case A & RES-Case B \\
\hline Spilled energy (TWh) & 0 & 0 & 0.1 \\
Spilled hours & 0 & 0 & 462 \\
Unserved energy (TWh) & 0 & 0 & 0.001 \\
Unmet hours & 0 & 0 & 6 \\
Electrical energy from GTs (TWh) & 18.73 & 23.56 & 31.73 \\
\hline
\end{tabular}

shows both improvement and deterioration in CCT following a three-phase fault on NSW/VIC interstate line for all the scenarios. Also, Figure 7 (b) demonstrates the speed of TPS-5 in p.u. before, during, and after a fault on SA/VIC interstate line with duration of 0.3 second at 10 p.m. of $18^{\text {th }}$ January 2020. As it is shown for this operating point, dynamical behaviour of the network is faster and the magnitude of the oscillation is amplified with higher penetration of intermittent RESs (i.e. due to less rotational inertia in the system).

2) Voltage stability: To calculate loadability, all loads in the network are scheduled to increase in small steps with constant power factor until the power flow fails to converge. Also, it is assumed that all the generators are scheduled with the same participation factor to pick up the system loads. The loadability of the system is computed for each hour from a step before the power flow divergence. Loadability results for all the scenarios are demonstrated in Figure 8. The BAU Scenario has the highest loadability followed by the RES-Case B and RES-Case A Scenarios. In the RES-Case B Scenario loadability is higher than RES-Case A Scenario. This is because more reactive power support is provided for the grid with higher generation from intermittent RESs (including converter-based WFs). Moreover, active power set-point for the critical generators (i.e. generators that limit loadability) is decreased allowing the system to undertake more demand. When CSP does not produce electrical energy (e.g. points $\mathrm{H}$ to K) loadability reduces. Also, when WFs generate low or high electrical energy, loadability declines as they cannot provide enough reactive power for the grid (e.g. area $\mathrm{A}$ to $\mathrm{C}$, and points $\mathrm{E}$ and $\mathrm{F}$ ). But, when their generation is between low and high (i.e. area D and point $G$ ), loadability improves. It is noteworthy to mention that in Figure 8 intermittent RESs are operating with PV control mode. Simulations results showed that if intermittent RESs operate with other control modes (e.g. PQ and droop), loadability does not improve (i.e. droop control mode) or even declines further (i.e. PQ control mode).

3) Frequency stability: Figure 9 illustrates the electrical frequency of TPS 4 in $\mathrm{Hz}$ when $2.2 \mathrm{GW}$ generation is lost in NSW at 10 p.m. of $18^{\text {th }}$ January 2020 . As it is shown in the figure, with less rotational inertia in the grid, output electrical frequency of generator drops deeper and faster. There is a steady-state error in the generator frequency because no automatic generation control (AGC) is considered in the system model. Also, due to better damping ratio for the renewable scenarios for that hour (Figure 6), oscillations damp quicker for renewable scenarios. Furthermore, steady-state frequency of TPS 4 is lower in the renewable scenarios in comparison with the BAU scenario.

\section{CONCLUSION}

In this paper, a simulation platform is presented for performance and stability assessment of FG scenarios. The platform

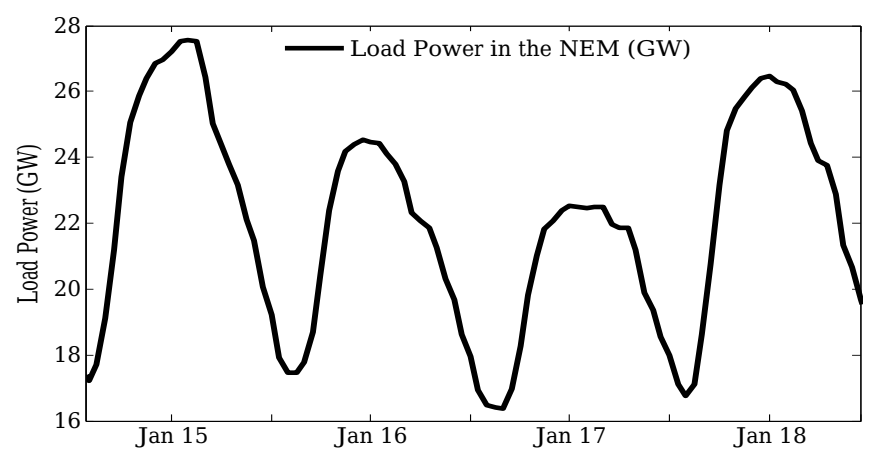

(a)

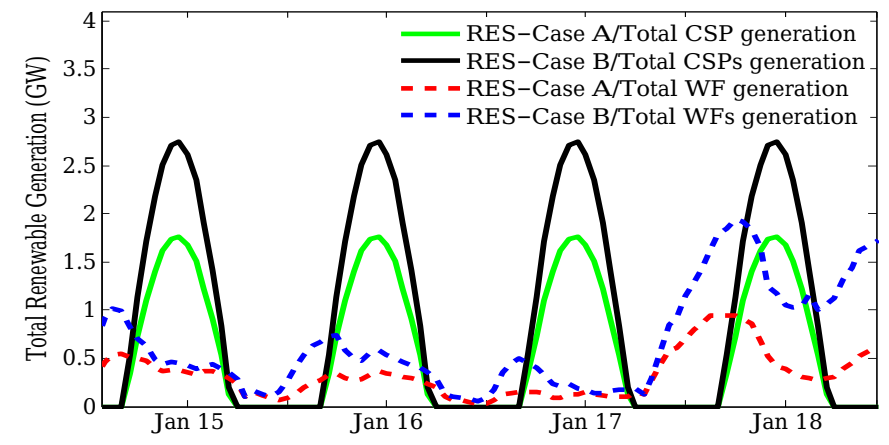

(b)

Fig. 5. (a) Demand profile, and (b) total RESs generation in the NEM

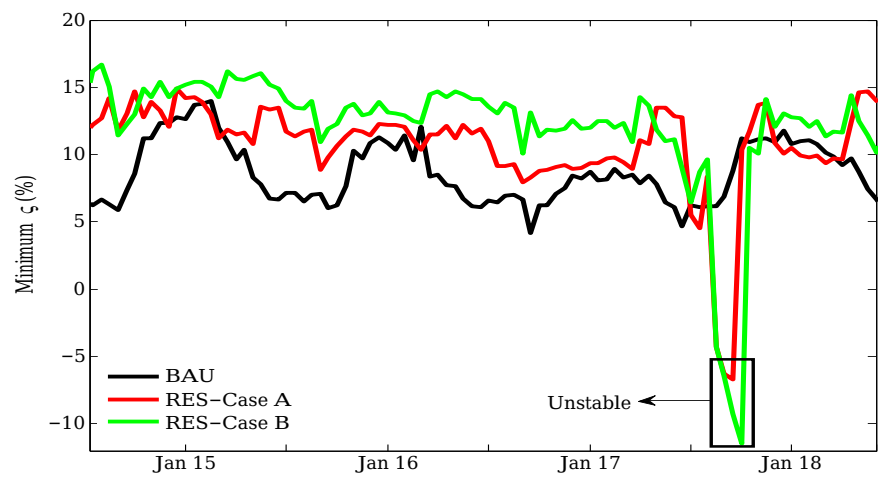

Fig. 6. Minimum damping ratio for all the scenarios

includes market simulation, load flow calculation and stability assessment together. Preliminary results for typical FG scenarios for the NEM in 2020 showed the importance of power system stability assessment. It is shown that with the increased penetration of WFs and CSPs in the grid, balancing between supply and demand will become more challenging, and more backup supply will be required. Also, it is illustrated that stability issues might change with the increased penetration of RESs in FGs. In terms of rotor angle stability (smallsignal and transient) both detrimental and beneficial impacts are observed. Voltage stability results showed that with low or high generation from WFs and/or no generation from CSPs, loadability deteriorates due to lack of reactive power support for the grid. Frequency stability results also demonstrate that displacing conventional generators with RESs, in particular inverter-based RESs, results in faster frequency dynamics because of less rotational inertia in the grid. 


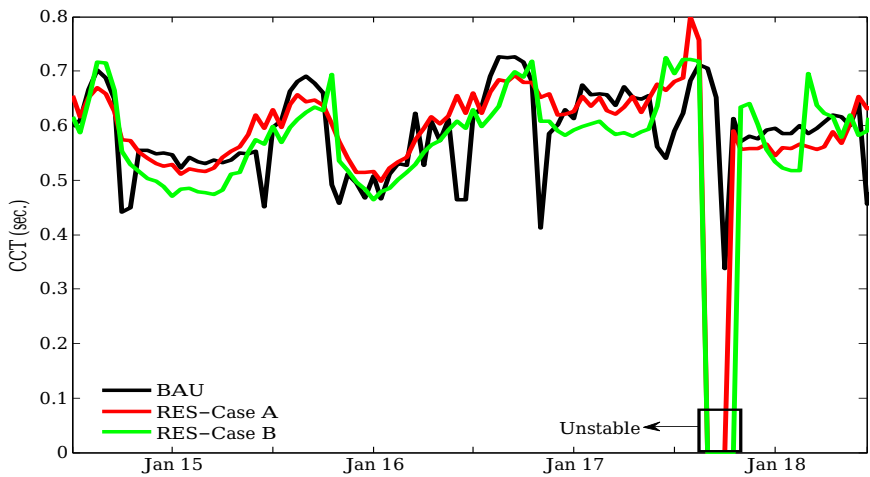

(a)

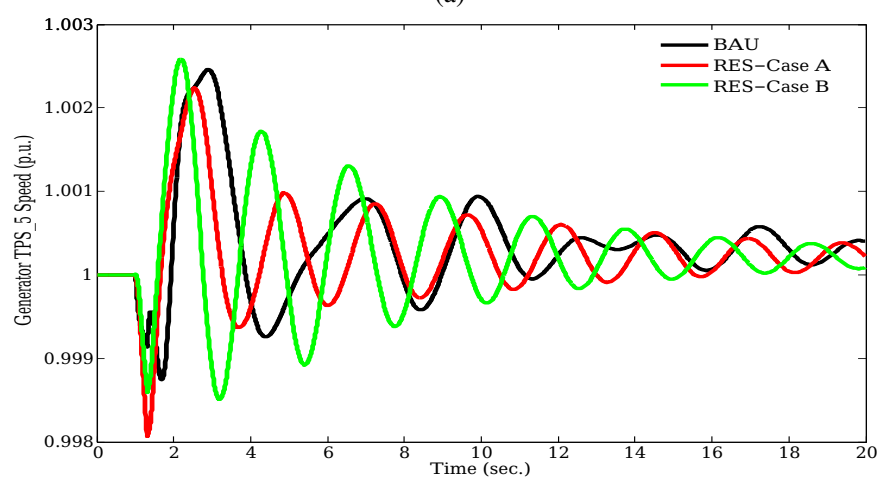

(b)

Fig. 7. (a) CCT following a three-phase fault on NSW/VIC line, and (b) Generator TPS-5 speed following a three-phase fault on SA/VIC line

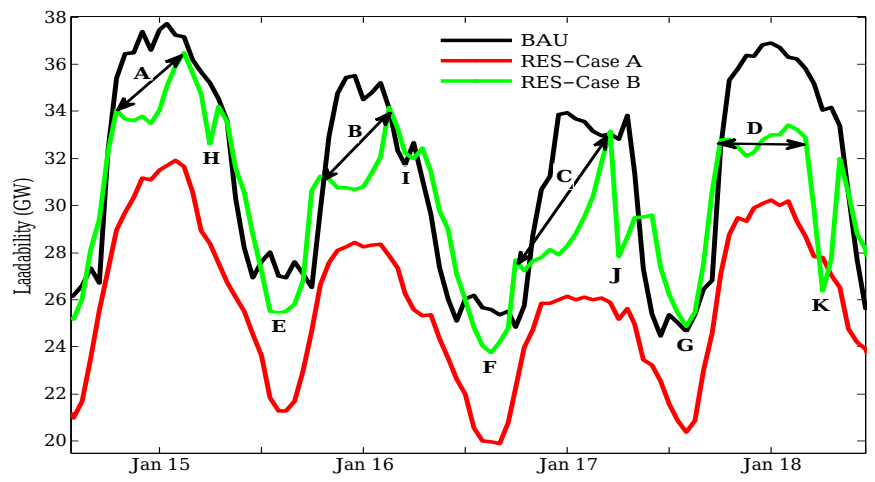

Fig. 8. Loadability results for all the scenarios

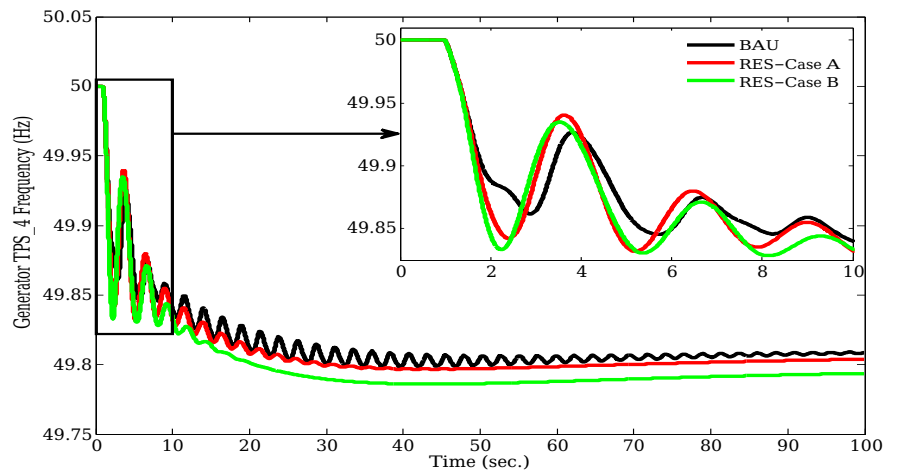

Fig. 9. Generator TPS_4 Frequency for all the scenarios
There is more to be explored in this area. The focus of future research is DR modelling for performance and stability assessment of FG scenarios. Also, for a general conclusion about the effect of RESs on the stability, it is necessary to study their effects using sensitivity analysis methods.

\section{REFERENCES}

[1] Australian Government, Department of Industry, "Energy facts, statistics and publications," 2014. [Online]. Available: http://www.innovation.gov.au/Energy/Pages/default.aspx

[2] Wright, M. and Hearps, P., "Zero Carbon Australia Stationary Energy Plan," The University of Melbourne Energy Research Institute, Tech. Rep., 2010.

[3] Elliston, B. and Diesendorf, M. and MacGill, I., "Simulations of Scenarios with $100 \%$ Renewable Electricity in the Australian National Electricity Market," Energy Policy, vol. 45, pp. 606-613, Jun. 2012.

[4] Elliston, B. and MacGill, I. and Diesendorf, M., "Least Cost 100\% Renewable Electricity Scenarios in the Australian National Electricity Market," Energy Policy, vol. 59, pp. 270-282, Aug. 2013.

[5] Budischak, C. and Sewell, D. and Thomson, H. and Mach, L. and Veron, D. E. and Kempton, W., "Cost-Minimized Combinations of Wind Power, Solar Power and Electrochemical Storage, Powering the Grid up to $99.9 \%$ of the Time," Journal of Power Sources, vol. 225, pp. 60-74, Mar. 2013.

[6] Hart, E. K. and Jacobson, M. Z., "A Monte Carlo Approach to Generator Portfolio Planning and Carbon Emissions Assessments of Systems with Large Penetrations of Variable Renewables," Renewable Energy, vol. 36, no. 8, pp. 2278-2286, Aug. 2011.

[7] REmap 2030, IRENA(2014), "REmap 2030; A Renewable Energy Roadmap," 2014. [Online]. Available: http://irena.org/remap

[8] Knorr, D.-I. K., "Abschätzung der Netzstabilität einer rein regenerativen Stromversorgung," in 17th Kasseler Symposium Enerie-Systemtechnik, 2012, pp. 1- 14 .

[9] Stock, S. and Faiella, M. and Lower, L. and Rohrig, K. and Hofmann, L. and Knorr, K., "Improving Grid Integration of Wind Energy Power Plants," Fraunhofer Institute for Wind Energy and Energy System Technology, Tech. Rep., 2012.

[10] Gibbard, M. and Vowles, D., "Simplified 14-Generator Model of the SE Australian Power System," The University of Adelaide, Tech. Rep., 2010.

[11] AEMO, "2012 NTNDP Assumptions and Inputs," 2012. [Online]. Available: http://www.aemo.com.au/Electricity/Planning/NationalTransmission-Network-Development-Plan/Assumptions-and-Inputs

[12] DIgSILENT GmbH, "DIgSILENT PowerFactory User Manual (Version 15.1)," DIgSILENT PowerFactory, Tech. Rep., 2013.

[13] Australian Government, Bureau of Resources and Energy Economics, “Australian Energy Technology Assessment," Tech. Rep., 2012.

[14] CSIRO FG Forum, "Future Grid Forum: change and choice for Australias electricity system," 2013. [Online]. Available: http://www.csiro.au/Organisation-Structure/Flagships/EnergyFlagship/Future-Grid-Forum-brochure.aspx

[15] Parkinson, G., "People power: Rooftop solar PV reaches 3GW in Australia," 2013. [Online]. Available: http://reneweconomy.com.au/2013/people-power-rooftop-solar-pvreaches-3gw-in-australia-99543

[16] Alvarado, F.L. and Meng, J. and DeMarco, C.L. and Mota, W.S., "Stability Analysis of Interconnected Power Systems Coupled with Market Dynamics," IEEE Transactions on Power Systems, vol. 16, no. 4, pp. 695-701, 2001.

[17] Kundur, P., Power System Stability and Control. EPRI Power System Engineering Series, McGraw-Hill, 1994.

[18] Tischer, H. and Verbic, G., "Towards a Smart Home Energy Management System-A Dynamic Programming Approach," in Innovative Smart Grid Technologies Asia, 2011, pp. 1-7.

[19] A. Chapman, G. Verbic, and D. Hill, "A Healthy Dose of Reality for Game-Theoretic Approaches to Residential Demand Response," in Bulk Power System Dynamics and Control - IX Optimization, Security and Control of the Emerging Power Grid (IREP), 2013 IREP Symposium. 